\title{
Mini-Review
}

Theme: Stability of Pharmaceutical Excipients

Guest Editors: S.Narasimha Murthy and Michael A. Repka

\section{Stability of Vaccines}

\author{
N. Dumpa, ${ }^{1}$ K. Goel, ${ }^{1}$ Yuhan Guo, ${ }^{1}$ H. McFall, ${ }^{1}$ Amit Raviraj Pillai, ${ }^{1}$ Ashay Shukla, ${ }^{1}$ \\ M. A. Repka, ${ }^{1}$ and S. Narasimha Murthy ${ }^{1,2}$
}

Received 1 June 2018; accepted 3 October 2018; published online 4 January 2019

Abstract. Vaccines are considered the most economical and effective preventive measure
against most deadly infectious diseases. Vaccines help protect around three million lives
every year, but hundreds of thousands of lives are lost due to the instability of vaccines. This
review discusses the various types of instability observed, while manufacturing, storing, and
distributing vaccines. It describes the specific stability problems associated with each type of
vaccine. This review also discusses the various measures adopted to overcome these
instability problems. Vaccines are classified based on their components, and this review
discusses how these preventive measures relate to each type of vaccine. This review also
includes certain case studies that illustrate various approaches to improve vaccine stability.
Last, this review provides insight on prospective methods for developing more stable
vaccines.

KEY WORDS: vaccine; stability; attenuated; protein; antigen.

\section{INTRODUCTION}

In general, a vaccine is a biological preparation that provides acquired immunity or adaptive immunity to a particular disease. According to the WHO (World Health Organization), vaccines can now be administered to prevent 25 different diseases. A typical vaccine contains an agent, which stimulates the body's immune system. This agent can be a weakened or inert form of the target pathogen, the toxins produced by the pathogen, or surface proteins of the pathogen.

Vaccination is the most economical method to prevent many infectious diseases that cause morbidity or mortality. However, the instability of vaccines limits their utilization in many developing countries where adequate storage facilities are unavailable. Most vaccines have poor thermostability; they require uninterrupted storage at $2-8^{\circ} \mathrm{C}$ from the manufacturing of the vaccine until it is administered to a patient. The storage of vaccines outside of this required temperature range is often due to poor infrastructure facilities, and this accounts for about $80 \%$ of the total cost of vaccination programs, which is around $\$ 200-\$ 300$ million

N. Dumpa, K. Goel, Yuhan Guo, H. McFall, Amit Raviraj Pillai, Ashay Shukla, M. A. Repka and S. N. Murthy contributed equally to this work.

\footnotetext{
${ }^{1}$ Department of Pharmaceutics and Drug Delivery, The University of Mississippi, University, MS 38677, USA.

${ }^{2}$ To whom correspondence should be addressed. (e-mail: murthy@olemiss.edu)
}

per year $[1,2]$. The development of vaccines that are stable for long periods of time will not only reduce their financial burden but also expand their accessibility to remote regions around the world. This would help prevent millions of deaths each year from infectious diseases that could be prevented by vaccines [3].

Vaccines are biological products, which can be briefly classified as (a) live, attenuated bacterial (LAB) vaccines; (b) subunit vaccines; (c) live, attenuated viral vaccines; (d) recombinant vaccines; (e) conjugated polysaccharide vaccines; (f) inactivated viral vaccines; and (g) combination vaccines. Each of these vaccines has its own stability issues, which must be considered during development. Due to their instability, they cannot be stored in the same way as chemical products. These stability problems hinder the safety and efficacy of the vaccine, and this loss of potency cannot be reversed. Vaccine instability can be caused by light, heat, radiation, changes in the environment, or reactions with the container or with other components in the mixture. Even when optimal conditions are maintained, potency may decline gradually as time passes after production. Optimizing the use of vaccines depends heavily on maintaining optimal storage conditions (e.g., $\left.2-8^{\circ} \mathrm{C}\right)$.

Maintaining this temperature range generally requires a cold chain. A cold chain is a temperature-controlled supply chain used to maintain a desired low-temperature range. The cold chain technique is a widely used process for the transportation of vaccines to various parts of the world. This process is frequently used with vaccines to maintain optimal temperatures that ensure the vaccine will not lose potency. 
An ideal cold chain consists of uninterrupted refrigeration of the vaccine through processes like production, storage, and even distribution. This manuscript discusses the stability issues of each vaccine type and provides insight on potential methods for overcoming these challenges.

\section{LIVE, ATTENUATED BACTERIAL VACCINES}

The oral administration of LAB vaccines has been successful due to their ability to imitate natural infections and elicit both mucosal and systemic immune responses [4, 5]. These vaccines have been developed for a number of diseases, such as anthrax, plague, cholera, dysentery, brucellosis, and Q fever; however, the bacillus Calmette-Guérin (BCG) and Ty21a oral typhoid vaccines are the two most widely used vaccines of these types.

Only a few LAB vaccines are commercially available due to concerns about their safety and their poor stability. LAB vaccines are commonly susceptible to instabilities caused by high residual moisture, extreme temperatures, ultraviolet radiation, and insufficient excipients [6,7]. It is important to monitor the stability of these vaccines throughout their formulation and manufacture.

Salmonella Typhi Ty21a was discovered in the early 1970s through random chemical mutagenesis using nitrosoguanidine, which resulted in GalE- and virulence (Vi)-negative phenotypes [8,9]. Random chemical mutagenesis has also led to other mutations in the Ty21a strain, and these mutations are believed to cause sensitivity to thermal, oxidative, and osmotic stresses [10]. Therefore, there is a need for controlled mutagenesis in order to have defined, attenuating mutations.

The characterization of these mutations has traditionally been determined by the phenotypes associated with attenuation, which poses a problem for controlled mutations. Also, this method is only an indirect way of evaluating instability. Kopecko et al. [10] used genetic tools to demonstrate that these phenotypes could be correlated with specific genetic mutations. These results are useful because a change in the genotype could be identified before a noticeable change in the phenotype can be observed [10]. This study indicates that genetic analysis methods could be implemented in the manufacturing process to directly validate the genetic stability of Ty21a.

\section{SUBUNIT VACCINES}

Subunit vaccines are prepared by extracting and purifying some components of the bacteria that can elicit an immunologic process [11]. Compared to traditional attenuated vaccines, subunit vaccines are safer as they circumvent the stimulation of immune system thereby reducing the risk of subsequent reversion, which can be caused by traditional attenuated microorganism vaccines [12] and less thermally sensitive $[11,12]$. However, these vaccines can fail to elicit an effective immune response [12], and these vaccines must be administered to patients in several doses at designated time intervals to ensure their long-term sufficiency [13].

The addition of adjuvants is needed in the vaccine formulation in order to foster an immunologic response. Adjuvants are substances that can be used as immuno- potentiators or delivery systems [14]. The most commonly used adjuvants are aluminum salts [15], but the mechanism behind their role in eliciting an immunological response remains controversial as Alum is believed to promote Th2type immune responses and differentiation of $\mathrm{B}$ cells resulting in robust antibody production. However, the role of $\mathrm{Th} 2$ cytokines in the adjuvant activity of alum is not clearly defined. In vitro studies indicate that alum-induced activation of macrophages and up-regulation of co-stimulatory molecules did not depend on IL-4, whereas in vivo studies suggest alum-induced priming of B cells through IL-4 producing Gr1+ cells in mouse spleen, which is required for proliferation of antigen-specific B cells and for optimal antibody production $[16,17]$. There is a need for further research on new adjuvants and more definitive mechanisms to ensure effective delivery systems and efficient immunological response [18]. Also, in order to optimize the characteristics of subunit vaccines, several components must be contained in a single vaccine, such as different antigens or adjuvants [17], which require thorough and comprehensive studies on formulation.

As drug delivery systems, adjuvants can improve antigen uptake, stabilize the antigens, and achieve sustained release $[16,19]$. Therefore, developing combination adjuvants as immunopotentiators and stabilizers is an ideal way to develop high-efficiency adjuvants [16]. However, combining adjuvants could potentially cause antagonism [20]. The safety of new adjuvants must be heavily researched, and adjuvants should be assessed both individually and after their combination. Many of the current adjuvants can cause side effects, such as local inflammation [21-24].

\section{Tetanus Toxoid}

The tetanus toxoid vaccine first appeared in 1890 after researchers had tried to eradicate tetanus for over a century with no success [25]. Some studies have shown that the tetanus toxoid vaccine was sensitive to freezing temperatures but not highly sensitive to heat [12]. One study showed that the tetanus toxoid vaccine could remain immunogenic and safe in a CTC (controlled temperature chain) up to $40^{\circ} \mathrm{C}$ for no more than 30 days [26].

The tetanus toxoid vaccine aggregates when moisture is absorbed, similar to other solid protein drugs. This aggregation can reduce the potency of the vaccine. However, a minimum amount of water is needed to maintain the $3 \mathrm{D}$ structure of the protein. Therefore, osmolytes must be added during the drying process of the protein [27, 28].

Jain and Roy [27] demonstrate that nucleic acid aptamers can serve as stabilizers for proteins. Jain and Roy lyophilized tetanus toxoid and specific aptamers, then encapsulated them in PLGA microspheres. After sustained release for 55 days, they found that the aptamers inhibited the protein from absorbing moisture, which inhibited aggregation [29].

Oral vaccinations are cheaper and safer in terms of needle-borne infections. Therefore, the development of oral vaccines is inevitable [30]. Harde et al. [25] developed a method that uses glutaraldehyde as a cross-linking agent to combine tetanus toxoid and sCh-GM-NPs (stabilized mannosylated chitosan nanoparticles) for oral vaccination. After in vitro stability studies and immunogenic studies in 
mice, they found that this stabilized nanoformulation exhibits the desired high stability as well as high mechanical strength in biological media. Harde et al. [25] concluded that sCh-GMNPs are a promising adjuvant for oral tetanus vaccinations.

\section{Anthrax}

Anthrax can infect humans in many ways, including skin contact and inhalation. Although the anthrax epidemic has been well controlled, occasional outbreaks of anthrax still pose a grave threat to human health due to its high lethality and its uncontrollable speed of dissemination. Therefore, it is important to produce an anthrax vaccine that is both effective and reliable. The stability of an anthrax vaccine often depends on its formulation.

Aluminum salts are the most commonly used adjuvants in anthrax vaccine formulations. Second-generation anthrax vaccines use a recombinant protective antigen (rPA) to elicit a strong immunologic response. Compared to the conventional anthrax vaccine (AVA), rPA vaccines are highly purified and can elicit an effective immunologic response with fewer doses [31-33]. However, rPA vaccines exhibit a thermal sensitivity, so extreme that instability issues arise even in refrigerated conditions [34].

Several studies demonstrate the improved stability of dried formulations [35-37]. According to these results, Jones et al. [34] used drying techniques to develop a plantreduced, deglycosylated rPA vaccine candidate (pp-dPA83) with a hydrogel as the adjuvant. After a series of stability tests, this vaccine remained immunogenic. Although this technique must be further researched, it is still a promising method for addressing thermal instabilities and eliminating the need for a CTC.

Since anthrax can be inhaled into the body, some scientists are developing nasal mucosal vaccination methods $[38,39]$. Nasal mucosal vaccinations can elicit both systemic immunity and mucosal immunity [40]. Wang et al. [37] used a mast cell activator compound $\mathrm{C} 48 / 80$ as an adjuvant in a mucosal vaccination administered by direct nasal delivery. They discovered that this is a promising method for developing stable and effective anthrax vaccines.

\section{LIVE, ATTENUATED VIRAL VACCINES}

Live, attenuated viral (LAV) vaccines are the most promising method for the prevention of various viral diseases, such as measles, mumps, yellow fever, polio (OPV), smallpox, and influenza (LAIV) [41]. They are exceptionally unstable because their infectivity and viability depend on their complex structure, their need to retain protein conformation, and the structure of their functional proteins [42]. It is estimated that about $50 \%$ of vaccines formulated by lyophilization are discarded annually due to the poor thermostability of LAV vaccines [2].

LAV vaccines are highly instable in aqueous formulations due to several chemical and physical reactions; hence, the majority of these vaccines are manufactured as dry formulations. Physical instabilities occur due to conformational changes. The unfolding of proteins increases unfavorable thermodynamic interactions that result in aggregation, precipitation, or adsorption of proteins, because the secondary, tertiary, and quaternary structural units of the proteins are altered. Chemical instabilities usually occur due to chemical alterations of the protein structure.

LAV vaccines lose potency due to chemical reactions that include hydrolysis, deamidation (alteration of amino acid side chains in asparagine residues), oxidation (cysteine and methionine residues), and alteration of disulfide bonds [4245]. Therefore, macromolecules are biologically active and more stable in the dry state due to decreased mobility and the absence of mechanisms that cause instability [46]. Many LAV vaccines, which do not contain stabilizing agents, are more heat sensitive, thus potency loss occurs during long-term storage in the solid state as well as shortly after their reconstitution.

\section{Oral Polio Vaccine}

Poliomyelitis is caused by any of the three poliovirus serotypes (types 1,2 , or 3 ). It predominantly affects children, and it cannot be treated. However, it can be prevented [47]. Two vaccines types are currently used for prevention of poliomyelitis: the inactivated polio vaccine (IPV), administered as an injection, or the attenuated polio vaccine (OPV), administered as oral drops.

Although both OPV and IPV are effective for preventing poliomyelitis, OPV is preferred over IPV because of its advantages, such as superior mimicry of natural infection for longer durations without adjuvants, ease of administration, and ease of manufacturing. However, OPV has some disadvantages. It requires a cold chain and can induce vaccine-associated poliomyelitis [42]. OPV must be frozen for long-term use or should be used within 30 days even when stored between 2 and $8{ }^{\circ} \mathrm{C}$.

The $\mathrm{pH}$ level of an OPV formulation also affects its stability. Optimizing the $\mathrm{pH}$ enhances the stability of all three strains of OPV at elevated temperatures. OPV can also be stabilized by excipients, including $\mathrm{MgCl}_{2}$, L-cysteine, dimethyl sulfoxide, lysine, and ethylene diamine [48, 49]. Some studies show that deuterium and heavy water also act as stabilizing agents in OPV formulations either alone or in combination with $\mathrm{MgCl}_{2}$ [50].

Lyophilized formulations of the polio vaccine have been unsuccessful, since they become less effective during the drying process [51]. Some researchers have studied the effect of high hydrostatic pressure (HPH) on the thermal stability of the OPV strains. Interestingly, HPH did not inactivate any of the three strains; instead, it actually increased the thermal stability of OPV strains at $37^{\circ} \mathrm{C}$. This suggests that the stated method displays potential to enhance vaccine stabilization, but further studies need to be conducted to ensure the reproducibility and versatility of the same [52].

\section{Seasonal Influenza}

Seasonal influenza is the most prevalent respiratory condition caused by the influenza virus. It is responsible for high levels of mortality, morbidity, and financial loss [53-55]. There are three types of influenza virus (A, B, C). Influenza vaccine development targets the type $\mathrm{A}$ and type $\mathrm{B}$ virus, since these two types cause respiratory infections. Periodic changes in the two main surface glycoproteins, hemagglutinin 
(HA) and neuraminidase (NA), result in antigenic shift and antigenic drift. Because of these changes, the existing influenza vaccine must be updated annually [56].

The three vaccines currently available for the prevention of influenza infection are live-attenuated influenza vaccines (LAIVs), trivalent inactivated vaccines (TIVs), and recombinant influenza vaccines based on recombinant HA. Although LAIVs are effective and have low manufacturing costs, they require lyophilization, which limits their use in developing countries [54].

The stability of LAIVs is affected by several factors, such as the type of strain, buffer type, and aggregation [57]. Flumist ${ }^{\circ}$, a licensed LAIV, is reportedly stable for only 18 weeks at $2-8{ }^{\circ} \mathrm{C}$. White et al. [58] developed a stable liquid formulation of LAIV containing $1 \%$ arginine, $0.5 \%$ RHSA (recombinant human serum albumin (HSA)), and an optimized sucrose phosphate glutamate buffer, which is stable for 1 year at $2-8{ }^{\circ} \mathrm{C}$.

Aqueous formulations of influenza vaccine are more prone to physical and chemical alterations, including denaturation, aggregation, and changes in glycoprotein HA conformation. High temperatures accelerate these physical and chemical changes, while low temperatures (below the freezing point of the vaccine) damage the antigen through ice formation and enhance solute concentration [58].

\section{MMR Vaccines}

Measles, mumps, and rubella vaccines (MMR vaccines) are considered some of the most unstable live vaccines for humans. Many MMR vaccine formulations currently fail to meet the minimum time requirement, established by the WHO, of 1 week at $37{ }^{\circ} \mathrm{C}$ [59]. Rimevax ${ }^{\circledR}$, produced by GlaxoSmithKline, is stable for 5 weeks at $37{ }^{\circ} \mathrm{C}$. However, Attenuvax ${ }^{\circledR}$, the commercially available preparation from Merck, in lyophilized form, is stable for 1 week at $37^{\circ} \mathrm{C}$.

In one study, Ohtake et al. [60] developed a thermally stable formulation that remained stable for 8 weeks at $37{ }^{\circ} \mathrm{C}$ using spray-drying. They used the sugars sucrose and trehalose as stabilizers to protect against stresses from processing conditions. These sugars also reduced degradation mechanisms (e.g., aggregation) by forming a glassy matrix. Other components that helped enhance stability include glycerol (to decrease molecular mobility) and Larginine (to decrease reactions between proteins). HSA is believed to enhance thermostability by increasing $T_{\mathrm{g}}$. A pH of 7 or lower was also found to enhance the stability of the MMR vaccine [60].

Edens et al. [61] developed a patient-friendly formulation of the MMR vaccine that can easily be administered by unskilled personnel through a microneedle patch, thus avoiding needlestick injury. In this study, the vaccine was coated onto microneedles as a thin patch that dries immediately and does not require reconstitution. This formulation has a stability profile of 1 week at $25{ }^{\circ} \mathrm{C}$, which meets the WHO minimum requirement of stability at $25{ }^{\circ} \mathrm{C}$ in the presence of a desiccant. Commercially available MMR vaccines can lose potency if stored above the maximum recommended temperature $\left(8^{\circ} \mathrm{C}\right)$ despite containing protein and salt stabilizers [60].
Zhang et al. [62] developed a thermostable MMR vaccine using silk proteins as a matrix. Silk is a biologically derived protein polymer with useful properties, such as biocompatibility, mechanical strength, and slow, controlled degradation. In this study, the MMR vaccine was encapsulated in silk films through both lyophilization and air-drying. The MMR vaccine formulated with the air-drying technique showed thermostability at 25,37 , and $45{ }^{\circ} \mathrm{C}$, which is superior to dry lyophilized formulations. Silk films retained $83.9 \%$ potency after 6 months of storage, while the lyophilized powder form retained only $74.5 \%$ potency. The proposed mechanisms for this enhanced thermostability are a high $T_{\mathrm{g}}$ $\left(178{ }^{\circ} \mathrm{C}\right)$, an extensive physical cross-linking that immobilizes bioactive molecules, and a hydrophobic nature that results in low moisture content [62].

\section{Other LAV Vaccines}

Modified vaccinia ankara (MVA) and human adenovirus type 5 (ADHu5) are both unstable at elevated temperatures. Alcock et al. [3] used the sugars trehalose and sucrose to stabilize attenuated poxvirus MVA and ADHu5. They formed a glass in which the molecules were immobilized by suspending both these vaccines in the solutions of sucrose and trehalose and slowly drying them on a support membrane. Both vaccines formulated by this method had high viral titer recovery and immunogenicity for 6 months at $45{ }^{\circ} \mathrm{C}$ [3]. Another study used ice recrystallization inhibitors (IRI, $\mathrm{N}$ octyl-gluconamide (NOGIc) and ornithine glycine glycine galactose (OGG-Gal) to stabilize three viral vectors: vaccinia virus (VV), vesicular stomatitis virus (VSV), and herpes virus (HSV-1) [63].

\section{RECOMBINANT VACCINES}

Recombinant vaccines are vaccines derived from DNA recombination technology. The manufacturing of recombinant vaccines involves two main steps. The first step is inserting DNA fragments, which can encode the desired antigens into bacterial, yeast, or mammalian cells. In the second step, antigens are expressed in cells, extracted, and purified from the cells. Compared to live, attenuated vaccines and purified vaccines, recombinant vaccines can either reduce the risk of virus and toxoids reversion or they can produce pure antigens in sufficient quantities [64].

Recombinant vaccines must be highly specific to incite apposite immune response. To ensure their efficacy different kinds of adjuvants may be added to recombinant vaccines and different delivery systems may be applied [64]. Common recombinant vaccine strategies include recombination of proteins, recombination of live vectors to produce antigens, and injecting naked DNA into an organism to incite an immune response [64].

\section{Hepatitis B}

Hepatitis B virus (HBV) vaccine [65] is an effective and desirable tool for blocking the global spread of hepatitis $B$ viral infection [66, 67]. Currently, many formulations of HBV vaccines are liquid and contain aluminum salts as adjuvants [12]. Their formulation is the primary determining factor in 
the stability of HBV vaccine during cold chain [12]. Some studies have shown that $\mathrm{HBV}$ vaccines are not highly heat sensitive. When exposed to $45^{\circ} \mathrm{C}$ for 1 week, or to $37{ }^{\circ} \mathrm{C}$ for 1 month, HBV vaccines still maintained immunogenicity, although they lost some of their reactogenicity [68]. However, Zapata et al. [69] illustrate that the noteworthy disadvantage of HBV vaccine is its sensitivity to freezing. The process of freezing and thawing elicits the aggregation of the aluminum salts, which may cause irritation at the injection site [12] and reduce the potency of the vaccine due to the increase in aluminum particle size [70].

Currently available HBV vaccines must be kept in the cold chain from manufacturing until administration [71]. According to a study by Jezek et al. [72], the thermal stability of HBV vaccines was improved by adding a phosphate buffer (20-100 mM, pH 4.6-7.0) because the surface chemistry of the aluminum adjuvants was changed by the phosphate. Braun et al. [73] show that propylene glycol and polyethylene glycol 300 prevented HBV vaccines from agglomeration during the freeze-thaw process. The colligative property of glycols contributed to the depression of the freezing point.

Another way to improve the stability of HBV vaccines in both elevated and subzero temperatures is to formulate the vaccine as a dry powder. However, the extreme conditions of the drying process used in this method may damage the proteins in the vaccines [74]. Tonnis et al. [74] used inulin and a sugar mixture of dextran and trehalose as two separate stabilizers to formulate HBV vaccines as a powder through spray freeze-drying. In this experiment, they tested the thermal stability of the $\mathrm{HBV}$ vaccine during spray-freezedrying process, freeze-thaw cycles, and at 20 and $60{ }^{\circ} \mathrm{C}$ storage conditions. ELISA results showed that in the presence of sugars, the spray-drying process almost has no effect on the antigenicity of HBsAg. The results also showed that the antigenicity of spray freeze-drying formulations that contain sugars remains intact after 10 freeze-thawing cycles. In addition, powder HBV vaccines formulated by this method can be stored at room temperature for 3 months or longer.

\section{Human Papilloma Virus}

There are about 40 types of human papilloma virus (HPV), which are known to cause genital infections. Around 13 of them may cause cervical cancer in women. Vaccination has been proven as the most effective method for blocking the infection of HPV. Currently, bivalent, quadrivalent, and 9valent HPV vaccines are available on the market. The most recent 9-valent HPV vaccines have been developed to vaccinate against HPV-6, -11, -16, -18, -31, -33, -45, -52, and -58 , and they can completely substitute bivalent or quadrivalent HPV vaccines [75].

The recombinant method is desirable for developing HPV vaccines. HPV vaccines can be developed through the recombination of L1 proteins, whose expression in vitro can lead to the formation of VLPs derived from each subtype [76]. Recombinant HPV vaccines have the advantage of being safer than traditional vaccines, but their drawback is poor immunogenicity, which cannot be ignored. HPV VLPs obtained from yeast are inherently unstable and tend to aggregate. The addition of adjuvants is required to enhance the immune response [77]. Therefore, the development of new adjuvants is crucial for making HPV vaccines more stable and protective.

Currently, aluminum salts are the most commonly used adjuvant for the recombinant HPV vaccine. There have been many attempts to optimize these adjuvants. Shi et al. [78] show that non-ionic surfactants can improve the stabilization of recombinant HPV vaccines. They used polysorbate 80 (PS80) as a surfactant and added PS80 and electrolytes to HPV VLP solutions. After testing and characterizing, they concluded that the presence of a non-ionic surfactant, together with adequate number of electrolytes, could significantly enhance the stability of recombinant HPV vaccines. This enhanced stability results because PS80 protects the surface of the VLP and electrolytes provide sufficient ionic strength. Both of these are important factors in stabilizing HPV vaccines [78].

Furthermore, other platforms have been used in developing HPV vaccines. Some studies report that peptide-based and protein-based vaccines have weak immunogenicity and are not effective in eliciting all types of immune responses $[79,80]$. Live vector-based vaccines are relatively unsafe [81]. Therefore, DNA vaccines have improved potency, safety, and stability.

\section{CONJUGATED POLYSACCHARIDE VACCINES}

The polysaccharide capsular antigens of various bacteria, such as Pneumococci, meningococci, S. Typhi, Haemophilus influenzae type $\mathrm{b}$, produce an effective serum antibody response when used as a vaccine. When a polysaccharide hapten covalently conjugates with a protein carrier, a humoral immune response can be observed. This serves as an example of response that possesses the properties of $\mathrm{T}$ cell dependent antigens. These types of vaccines have been manufactured with four different protein carriers that vary in structure and conformation. These vaccines are administered using a very specific timeline: three doses during infancy and a fourth dose at 2 years.

In polysaccharides, DNA bases are linked to a deoxyribose backbone or carbohydrate monomers by glycosidic bonds [82, 83]. These bonds are affected by $\mathrm{pH}$, so these vaccines exhibit a minimum loss of activity between $\mathrm{pH} 6$ and 8. Aluminum salt adjuvants use electrostatic synergy to bind to specific antigens. Therefore, opposite charges between antigen and adjuvant are required for optimal formulation. Ionic strength also has a significant effect on the stability of these vaccines.

Debye-Huckel show that at comparatively low ionic strength $(I<0.15)$ with higher salt concentrations, biomolecular solubility decreased [84]. Some anions and cations display chaotropic effects, which can inflate macromolecular solubility and can affect both intermolecular and intramolecular stability. These effects are often caused by the involvement of water solvent as well as certain binding effects. Overall, ionic strength and $\mathrm{pH}$ both affect the stability of these vaccines.

Hypervirulent strains of Neisseria meningitidis, or the ET37 complex, were continually increasing during late 1990s. Three other conjugate vaccines that were developed are two conjugates to CRM197 (nontoxic mutant diphtheria toxins) and one conjugate to tetanus toxoid [85-92]. 


\section{INACTIVATED VIRAL VACCINES}

Inactivated viral vaccines have been developed for a number of diseases, such as influenza, hepatitis A, rabies, polio, and encephalitis. Inactivated viral vaccines are injectable and are administered intramuscularly in some vaccines. Formaldehyde and $\beta$-propiolactone (BPL) are widely used for the inactivation of licensed human viral vaccines for decades. Inactivated vaccines are generally more stable during long-term storage and are typically developed as liquid formulations stored in glass vials and prefilled syringes. However, some these vaccines can be sensitive to freezing and susceptible to potency loss during storage and distribution.

\section{Inactivated Poliomyelitis Vaccine}

The most widely used oral poliovirus vaccine (OPV), which is a live attenuated vaccine, has proven to be less effective and less safe than inactivated poliovirus vaccine (IPV) [93-95]. Therefore, in some developed countries, IPV has replaced OPV due to its more reliable safety and desirable potency to eliminate vaccine-derived poliomyelitis [95, 96].

Currently, the commercially available IPV vaccines are formulated as liquids, and most of these can maintain stability and potency in the cold chain up to 3 years. However, they not only are sensitive to freezing but also lose potency quickly when exposed to temperatures above room temperature $25{ }^{\circ} \mathrm{C}[43,97]$. Therefore, better formulations are needed to improve the thermal stability of IPV. Qi et al. [98] show that many stabilizers, such as disaccharides and sodium citrate, enhanced the thermal stability of IPV3. $\mathrm{D}_{2} \mathrm{O}$ can protect poliovirus virion from swelling or aggregation, making IPV more stable [98].

Furthermore, Tzeng et al. [99] developed a microsphereencapsulated IPV formulation. IPV showed better stability in higher temperatures after incubation with carbohydrate, $\mathrm{MgCl}_{2}$, and MSG (monosodium glutamate). IPV was encapsulated into PLGA-based microspheres, which were protected by the excipients. When these microspheres were developed as dry formulations, they were more stable than the liquid formulations [99]. Kraan et al. [100] changed the delivery route of IPV and showed that IPV can be formulated as a bioneedle, which are small hollow mini implants fabricated from biodegradable polymers that can be filled with antigen. Bio needles can be used for vaccination without syringes and needles. IPV was filled into bio-needles and lyophilized with the aid of excipients. This IPV formulation also showed greater heat resistance compared to the liquid IPV formulation. This IPV formulation is promising because it does not rely on the cold chain due to the nature of the bio-needles. However, this technique needs further research [100].

\section{COMBINATION VACCINES}

Combination vaccines are used to vaccinate against multiple diseases. They were initially used in 1948 to vaccinate infants and children. Individual diphtheria, tetanus, and pertussis (DPT) vaccines were combined into a single product commonly called DTP [101]. Various approaches have tried to improve the reactogenicity profile of DTP, including the addition of other vaccines and the removal of certain components.

The replacement of whole cell pertussis with less reactogenic acellular pertussis was an important step taken in the early 1990s to improve the reactogenicity profile. This advancement helped combine other vaccines, such as the $H$. influenzae vaccine (Hib), the IPV, and the hepatitis B vaccine $(\mathrm{HepB})$ to the existing DTP vaccine, which consisted of diphtheria, tetanus, and acellular pertussis antigens [102].

The MMR vaccine is another combination vaccine, but unlike the DTaP combination, no additional vaccines have been included over the years. The MMR vaccine has remained trivalent and is being administered as a single product [102].

Evaluating the stability of combined vaccines is difficult due to their complexity. The individual vaccines have their own specific stability issues that must be overcome first. Then, the vaccine components must maintain their stability after they are combined, and they must be compatible with each other.

The DTaP-IPV vaccine consists of the following eight antigens: diphtheria toxoid, tetanus toxoid, pertussis toxoid, filamentous haemaglutinin, pertactin, and inactivated poliovirus antigen types $1-3$. This vaccine requires multiple stability studies to determine the shelf life of intermediates and the final product expiration date [103]. The WHO states that the shelf life of the combined vaccine should be determined by the component with the shortest shelf life [104].

The stability problems of combination vaccines depend on complex interactions with various components. The pertussis antigens, diphtheria toxoid, and the hepatitis B antigen compete with each other for the binding sites on the adjuvant, and this competition often leads to a reduction in the potency of the diphtheria toxoid as the other antigens displace it.

Freeze-drying complex aluminum-absorbed preparations also leads to stability problems, especially for the Hib components [6]. A commonly reported immune interference occurs when combining the Hib component to the DTP vaccine, as it reduces antibody titers to the Hib component of the polyribosylribitol phosphate antigen [105-107]. This problem has also been reported in the hexavalent vaccine DTaP-HBV-IPV/Hib [108, 109]. This problem has not been observed to the same extent in the DTP vaccine, which has been credited to the adjuvant effect of the whole-cell pertussis component [105, 110, 111].

The adjuvant effect simply masks the underlying interference; hence, the mechanism by which Hib response is reduced when combined with DTaP is still not completely understood. Incompatibility with the aluminum adjuvant is another possible explanation for the reduction in immune response.

\section{CASE STUDIES}

\section{Typhoid Vaccine}

The commercial typhoid vaccine (Vivotif®) is a live attenuated vaccine for oral administration only in form an enteric-coated capsule formulation that has been lyophilized. The stability of this product is dependent on processing 
conditions, shelf-life, temperature, residual water, and excipients [7]. Freeze-drying and spray-drying are two common methods employed to stabilize solid formulations, but both methods expose the vaccine to extreme temperatures. Foamdrying has been used to perform the drying process at room temperature.

Ohtake et al. [7] conducted a study to evaluate the differences in process loss and storage loss for the three drying methods mentioned previously. The process losses for freeze-drying, spray-drying, and foam-drying are $0.8 \log _{10}, 0.7$ $\log _{10}$, and $0.3 \log _{10} \mathrm{CFU}$, respectively. The storage losses after 1 week at $25{ }^{\circ} \mathrm{C}$ for freeze-drying and spray-drying were 0.5 $\log _{10}$ and $1.7 \log _{10} \mathrm{CFU}$, respectively. The foam-drying samples showed no loss at these storage conditions [7]. Overall, foam-drying reduced the process loss and improved storage stability. This drying process was then used to evaluate the stability of Ty21a as it relates to growth media and conditions and formulation development.

The bacteria were harvested in the log and stationary phases for comparison. The theory is that the growth stages may have phenotypic differences, resulting in different levels of resistance to dehydration stresses [7]. The amount of bacteria recovered after the drying process was higher for the stationary phase. Next, the bacteria were grown with $\mathrm{NaCl}$, an osmotic stress-inducing agent, to determine whether this environment affected growth media or kinetics. As the concentration of $\mathrm{NaCl}$ increased, the bacteria exhibited a slower growth rate. In terms of growth kinetics, there is no advantage in using an osmotic stress-inducing agent.

The recovery of the bacteria after foam-drying and storage at $25{ }^{\circ} \mathrm{C}$ up to 16 weeks was then determined for the varying $\mathrm{NaCl}$ concentrations. The bacteria in the stationary phase showed increased process recovery for all concentrations of $\mathrm{NaCl}$. The highest survival rate was at a $\mathrm{NaCl}$ concentration of $0.3 \mathrm{M}$ in the stationary phase [7]. However, the $\mathrm{NaCl}$ concentrations showed no differential effect on the survival rates of the bacteria harvested in the log phase. Therefore, Ohtake et al. conclude that these results indicate that the addition of $\mathrm{NaCl}$ benefits Ty21a by potentially improving its tolerance to desiccation. Each of these studies indicates that the growth media and the particular phase may have an effect on the physical stability of the bacteria.

The second part of this study evaluates the effects of excipients on the stability of Ty21a. Methionine is a natural amino acid that is typically used as an antioxidant [7]. Its stabilizing effect was evaluated for formulations with concentrations ranging from 0.5 to $2 \%(w / v)$. They were stored for 8 weeks at $37^{\circ} \mathrm{C}$ with a $\mathrm{pH}$ of 8 [7]. As the concentrations increased, the storage stability actually decreased. The optimal concentration of methionine was $0.5 \%(w / v)$. The addition of plasticizers and even sugar solutions has shown improved storage stability after dehydration. The plasticizers, DMSO, and glycerol with concentrations from 0 to $2 \%(w / v)$ were shown to increase the process loss over 4 weeks at $37{ }^{\circ} \mathrm{C}$. However, DMSO was more successful at stabilizing the bacteria than glycerol, and its optimal concentration was $1 \%(w / v)$.

DMSO and methionine were then evaluated for their combined stabilizing effects. Methionine was maintained at $0.5 \%(w / v)$, while the concentration of DMSO was varied from 0 to $2 \%(w / v)$. The recovery of bacteria was calculated after being stored for 4 weeks at $37{ }^{\circ} \mathrm{C}$. DMSO at $1 \%(w / v)$ combined with methionine was found to be the optimal concentration for minimizing the process loss.

Last, the addition of gelatin to the formulations was evaluated. Although gelatin is commonly used as a stabilizer, it was incorporated in this study to minimize the rate of cavitation from drying by affecting the viscosity of the solution. Formulations were developed with or without gelatin, and each formulation contained either methionine, DMSO, or glycerol. All formulations also contained trehalose. The formulations were stored for 12 weeks at $37^{\circ} \mathrm{C}$. The addition of gelatin reduced the loss of bacteria for all formulations, but the formulation that contained methionine had the best survival rate. The formulation with trehalose, methionine, and gelatin were also stable for 12 weeks at $4{ }^{\circ} \mathrm{C}$ and at $25{ }^{\circ} \mathrm{C}$ [7]. These studies show how different types of excipients have different stabilizing effects on the storage stability of Ty21a. Therefore, formulation development should be optimized to improve the stability of vaccines.

The stability of the typhoid vaccine is also strongly dependent on $\mathrm{pH}$ and temperature. One technique for determining the effect of $\mathrm{pH}$ and temperature on cell viability is the use of a fluorescence-based analysis system, the LIVE/DEAD ${ }^{\circledR}$ BacLight ${ }^{\mathrm{TM}}$ bacterial viability kit [112]. This technique is based on the different permeabilities of intact versus damaged cell membranes. The SYTO® 9 (a greenfluorescent dye) is permeable to both intact and damaged membranes. Propidium iodine (PI, a red-fluorescent dye) can only permeate the damaged cells and will displace the green dye in those cells. The fluorescence emission intensity (CPS) is then plotted against the wavelength of light emitted. Peaks at $510 \mathrm{~nm}$ are attributed to the SYTO® 9 dye, and peaks at $600 \mathrm{~nm}$ are attributed to the PI.

When the Ty21a cell $\mathrm{pH}$ is 7 , a negligible PI peak was observed for temperatures of $10-30{ }^{\circ} \mathrm{C}$, indicating the presence of intact membranes. However, when the temperature was increased to $40{ }^{\circ} \mathrm{C}$ or greater, the intensity of the SYTO® 9 was greatly reduced and became similar to the PI peak. For Ty21a cells at a $\mathrm{pH}$ of 4 , the intensity was lower, and a PI peak was visible for all temperatures. These results demonstrate that more acidic environments and higher temperatures result in ruptured cell membranes, which in turn leads to a lower stability of Ty21a at these conditions [112].

Far-UV circular dichroism (CD) spectroscopy is another method used to characterize the changes in Ty21a when the $\mathrm{pH}$ and temperatures are varied. This technique showed the alterations in the protein structures in Ty21a cells. On the Far-UV spectra, the curves have two minima around 208 and $222 \mathrm{~nm}$ for $\mathrm{pH} 6,7$, and 8, which indicates an $\alpha$-helix secondary structure. At a $\mathrm{pH}$ of 5, the $\mathrm{CD}$ signal decreased to $208 \mathrm{~nm}$, and the absolute minima shifted to around $225 \mathrm{~nm}$. Further structural change is visible for $\mathrm{pH} 4$ with an even lower CD signal and right-shifted absolute minima. This demonstrates a considerable difference in protein structure compared to the neutral $\mathrm{pH}$ samples [112]. These changes in protein structure may be correlated with the membrane damage observed in more acidic $\mathrm{pH}$ environments.

An empirical phase diagram (EPD) is a tool that simplifies and compiles data for different techniques. An EPD can facilitate excipient screening and formulation development by providing necessary conditions for cell 
stability. An EPD was constructed for Ty21a cells using the data from the analyses of the effects of $\mathrm{pH}$ and temperature on the stability of these cells. The diagram plots temperature and $\mathrm{pH}$ on its axes, and it is color-coded, with each color signifying a different physical state of the Ty21a cells. Changes in these colors show modifications in the physical state of the cells.

The EPD for Ty21a has four distinct colors, which indicate at least four phases. In phase I, considerable cell membrane damage occurs for $\mathrm{pH} 4$ and 5 across all temperatures and for $\mathrm{pH} 6$ and 7 at temperatures greater than $30{ }^{\circ} \mathrm{C}$. Ty21a cells displayed the greatest viability in phase II, with the following parameters: pH 6 and 7 at 10$30{ }^{\circ} \mathrm{C}$ and $\mathrm{pH} 8$ at $10-25^{\circ} \mathrm{C}$. The conditions for phase III are $\mathrm{pH} 8$ for $25-45{ }^{\circ} \mathrm{C}$, in which cells are partially damaged. Last, phase IV is characterized by $\mathrm{pH} 8$ and temperatures greater than $45^{\circ} \mathrm{C}$, in which cells are even more severely damaged [112].

The drying methods, bacterial growth conditions, and excipients each affected the storage stability of Ty21a. For each of these factors, the process conditions and formulations must be optimized for better stability. The stability of Ty21a also depends on the $\mathrm{pH}$ and temperature. The EPD can be helpful in formulation development by summarizing the optimal conditions for this bacterial strain. These different instabilities of Ty21a are common to other live, attenuating bacterial vaccines.

\section{DTP Vaccine}

The DTP vaccine is one of the most widely used vaccines in immunization programs for children [113]. Other vaccines in combination with DTP include inactivated poliovirus, hepatitis B, or both. The diphtheria and tetanus toxoids, however, are the most stable components of these vaccines; they remain stable for $2.5-6$ years. The stability of the pertussis component is less stable, and there is limited information on this vaccine due to complicated and expensive potency tests [113]. Extreme temperatures and aluminum adjuvants affect the stability of the diphtheria and tetanus components. The pertussis vaccine is affected by temperature, whether it is monovalent or a combination, and the nature of the preservative or adjuvant [114].

Diphtheria and tetanus have been shown to remain stable at higher temperatures for longer periods of time. The toxoid components show little to no loss of potency when stored at $18{ }^{\circ} \mathrm{C}$ for 1.5 years, at $24{ }^{\circ} \mathrm{C}$ for $6-12$ months, and at $37{ }^{\circ} \mathrm{C}$ for $2-6$ months. Even at $45{ }^{\circ} \mathrm{C}$ for $2-4$ weeks, the toxoids showed no significant loss of potency. However, by week 8 at $45{ }^{\circ} \mathrm{C}$, the loss of potency had decreased by $40 \%$. Above $45{ }^{\circ} \mathrm{C}$, the protein-based toxoids denatured rapidly and no longer followed Arrhenius behavior. The toxoids will even be destroyed in $3-5 \mathrm{~h}$ when exposed to $60{ }^{\circ} \mathrm{C}$. The recommended shelf life temperature for these components is $2-8{ }^{\circ} \mathrm{C}$, but unintentional exposure to elevated temperatures is inevitable. Therefore, based on the above stability studies, Galazka et al. [114] determine that these toxoids can be stored at room temperature for up to 2 weeks without the need to test for potency.

The above studies show that tetanus toxoids are relatively stable at elevated temperatures, but they are more susceptible to loss of potency at freezing temperatures. In animal potency testing, which is a challenge based study wherein vaccinated and unvaccinated individuals may be compared after direct challenge with the target pathogen under controlled experimental conditions, the composition of the vaccine varied the reductions in potency when the tetanus toxoid was exposed to low temperatures [114]. In one study, five DTP vaccines were exposed to $-30{ }^{\circ} \mathrm{C}$ for $12 \mathrm{~h}$, and the tetanus component in two of these vaccines exhibited a potency loss of around $30 \%$. No significant loss of potency was observed when these vaccines were exposed to temperatures between -5 and $-10{ }^{\circ} \mathrm{C}$. However, in the DT vaccine, the tetanus compound showed a potency loss at both -5 and $-30{ }^{\circ} \mathrm{C}$. It is obvious that the adjuvant effect in the pertussis component causes this difference, but the reason for this remains unknown.

Both elevated and freezing temperatures affect the pertussis vaccine. When stored at $4-6{ }^{\circ} \mathrm{C}$ for 2 years, the pertussis component in the DTP and DTP-polio vaccines maintained a satisfactory level of potency. However, even under optimal conditions, the vaccine still showed a continuous loss of potency. The pertussis component retained $80 \%$ of its initial potency level for $2-8$ weeks at $22-25^{\circ} \mathrm{C}$. After this time, the loss of potency was around $0.3-0.4 \%$ per day. The loss of potency was about $10 \%$ within the first days at $45-$ $46{ }^{\circ} \mathrm{C}$. Then after 4-7 days, the loss of potency was about $50 \%$ of the original value at this temperature. Once the temperature reaches approximately $50-56{ }^{\circ} \mathrm{C}$, the loss of potency is rapid and complete [114].

The effect of freezing temperatures on the potency of the pertussis component was even more pronounced than the effect of elevated temperature. The potency decreases by about $50 \%$ when the vaccine was stored at $-20{ }^{\circ} \mathrm{C}$ for 15 days. In one study, three out of five manufactured vaccines had a significant loss of potency at -5 to $-10{ }^{\circ} \mathrm{C}$ and from 20 to $-30{ }^{\circ} \mathrm{C}$ [114]. These studies demonstrate how extreme temperatures affect the stability of the pertussis component in DTP vaccines.

Another factor that affects the stability of the pertussis vaccine is whether it is in monovalent form or combined with another vaccine. Studies show that monovalent pertussis vaccine is unstable at $4{ }^{\circ} \mathrm{C}$, with some samples losing 58 $87 \%$ of their potency in 18 months. B. pertussis bulk suspensions have more rapid rates of deterioration when stored for 1 year at $4{ }^{\circ} \mathrm{C}$ compared to pertussis in DTP [114]. Preservatives and adjuvants also have an effect on the stability of pertussis. If DTP-polio is preserved with benzethonium chloride (BC), the pertussis component loses a significant portion of its potency. One study preserved the pertussis vaccine with $\mathrm{BC}$ at $37{ }^{\circ} \mathrm{C}$ for 16 weeks. After 5 weeks, the vaccine potency had decreased by almost $50 \%$. At the end of the 16 weeks, the pertussis vaccine treated with $\mathrm{BC}$ had no measurable protection potency [114].

The stability issues of individual vaccines must be addressed first before the stability and compatibility of the combined vaccines can be evaluated. The DTP vaccine is one of the most common combined vaccines used globally. The tetanus and diphtheria components are more stable than the pertussis components. Extreme temperatures affect the stability of all three components. Other stability factors include adjuvants, preservatives, and the composition of the 
vaccine. Overall, it is difficult to conduct stability studies on combined vaccines due to their extreme complexity.

\section{CONCLUSION}

Vaccines have had a significant effect on medicine. They have also significantly affected society as a proven and powerful tool for public health. They provide protection against multiple diseases, but they also have stability problems, such as poor thermostability and adverse reactions to adjuvants. In the short term, overcoming these stability issues will help vaccines become more accessible to developing nations that have limited supplies of stable vaccines. In the long term, due to the importance of vaccines, overcoming these stability problems will be a major step toward the future of medicine.

Publisher's Note Springer Nature remains neutral with regard to jurisdictional claims in published maps and institutional affiliations.

\section{REFERENCES}

1. Rexroad J, Wiethoff CM, Jones LS, Middaugh CR. Lyophilization and the thermostability of vaccines. Cell Preserv Technol. 2002;1:91-104.

2. Wang G, Li X, Mo L, Song Z, Chen W, Deng Y, et al. Eggshell-inspired biomineralization generates vaccines that do not require refrigeration. Angew Chem. 2012a;124:10728-31.

3. Alcock R, Cottingham MG, Rollier CS, Furze J, De Costa SD, Hanlon M, et al. Long-term thermostabilization of live poxviral and adenoviral vaccine vectors at supraphysiological temperatures in carbohydrate glass. Sci Transl Med. 2010;2:19ra12.

4. McGhee JR, Mestecky J, Dertzbaugh MT, Eldridge JH, Hirasawa M, Kiyono $\mathrm{H}$. The mucosal immune system: from fundamental concepts to vaccine development. Vaccine. 1992;10:75-88.

5. Shata MT, Stevceva L, Agwale S, Lewis GK, Hone DM. Recent advances with recombinant bacterial vaccine vectors. Mol Med Today. 2000;6:66-71.

6. Corbel MJ. Reasons for instability of bacterial vaccines. Dev Biol Stand. 1996;87:113-24.

7. Ohtake S, Martin R, Saxena A, Pham B, Chiueh G, Osorio M, et al. Room temperature stabilization of oral, live attenuated Salmonella enterica serovar Typhi-vectored vaccines. Vaccine. 2011;29:2761-71.

8. Germanier R, Fuer E. Isolation and characterization of Gal E mutant Ty 21a of Salmonella Typhi: a candidate strain for a live, oral typhoid vaccine. J Infect Dis. 1975;131:553-8.

9. Germanier R, Fürer E. Characteristics of the attenuated oral vaccine strain "S. Typhi" Ty 21a. Dev Biol Stand. 1982;53:3-7.

10. Kopecko DJ, Sieber H, Ures JA, Furer A, Schlup J, Knof U, et al. Genetic stability of vaccine strain Salmonella Typhi Ty21a over 25 years. Int J Med Microbiol. 2009;299:233-46.

11. Moyle PM, Toth I. Self-adjuvanting lipopeptide vaccines. Curr Med Chem. 2008;15:506-16.

12. Kumru OS, Joshi SB, Smith DE, Middaugh CR, Prusik T, Volkin DB. Vaccine instability in the cold chain: mechanisms, analysis and formulation strategies. Biologicals. 2014;42:237-59.

13. Demento SL, Siefert AL, Bandyopadhyay A, Sharp FA, Fahmy TM. Pathogen-associated molecular patterns on biomaterials: a paradigm for engineering new vaccines. Trends Biotechnol. 2011;29:294-306.

14. Foged C, Hansen J, Agger EM. License to kill: formulation requirements for optimal priming of $\mathrm{CD} 8(+) \mathrm{CTL}$ responses with particulate vaccine delivery systems. Eur J Pharm Sci. 2012;45:482-91.

15. Lindblad EB. Aluminium adjuvants-in retrospect and prospect. Vaccine. 2004;22:3658-68.

16. Mutwiri G, Gerdts V, Littel v D, van den Hurk S, Auray G, Eng N, et al. Combination adjuvants: the next generation of adjuvants? Expert Rev Vaccines. 2011;10:95-107.

17. Pulendran B, Ahmed R. Immunological mechanisms of vaccination. Nat Immunol. 2011;12:509-17.

18. Oyston P, Robinson K. The current challenges for vaccine development. J Med Microbiol. 2012;61:889-94.

19. McNeela E, Lavelle E. Recent advances in microparticle and nanoparticle delivery vehicles for mucosal vaccination, mucosal vaccines. Berlin: Springer; 2011. p. 75-99.

20. Ghosh TK, Mickelson DJ, Solberg JC, Lipson KE, Inglefield JR, Alkan SS. TLR-TLR cross talk in human PBMC resulting in synergistic and antagonistic regulation of type- 1 and 2 interferons, IL-12 and TNF-alpha. Int Immunopharmacol. 2007;7:1111-21.

21. Petrovsky N, Aguilar JC. Vaccine adjuvants: current state and future trends. Immunol Cell Biol. 2004;82:488-96.

22. Reed SG, Orr MT, Fox CB. Key roles of adjuvants in modern vaccines. Nat Med. 2013;19:1597-608.

23. Leleux J, Roy K. Micro and nanoparticle-based delivery systems for vaccine immunotherapy: an immunological and materials perspective. Adv Healthc Mater. 2013;2:72-94.

24. Sahdev P, Ochyl LJ, Moon JJ. Biomaterials for nanoparticle vaccine delivery systems. Pharm Res. 2014;31:2563-82.

25. Harde H, Agrawal AK, Jain S. Tetanus toxoids loaded glucomannosylated chitosan based nanohoming vaccine adjuvant with improved oral stability and immunostimulatory response. Pharm Res. 2015;32:122-34.

26. Juan-Giner A, Domicent C, Langendorf C, Roper MH, Baoundoh P, Fermon F, et al. A cluster randomized noninferiority field trial on the immunogenicity and safety of tetanus toxoid vaccine kept in controlled temperature chain compared to cold chain. Vaccine. 2014;32:6220-6.

27. Jain NK, Roy I. Accelerated stability studies for moistureinduced aggregation of tetanus toxoid. Pharm Res. 2011;28:626-39.

28. Jiang W, Schwendeman SP. Formaldehyde-mediated aggregation of protein antigens: comparison of untreated and formalinized model antigens. Biotechnol Bioeng. 2000;70:507-17.

29. Jain NK, Jetani HC, Roy I. Nucleic acid aptamers as stabilizers of proteins: the stability of tetanus toxoid. Pharm Res. 2013;30:1871-82.

30. Jain S, Harde H, Indulkar A, Agrawal AK. Improved stability and immunological potential of tetanus toxoid containing surface engineered bilosomes following oral administration. Nanomedicine. 2014;10:431-40.

31. Powell AJ, Little CB, Hughes CE. Low molecular weight isoforms of the aggrecanases are responsible for the cytokineinduced proteolysis of aggrecan in a porcine chondrocyte culture system. Arthritis Rheum. 2007;56:3010-9.

32. Verma R, Oania RS, Kolawa NJ, Deshaies RJ. Cdc48/p97 promotes degradation of aberrant nascent polypeptides bound to the ribosome. Elife. 2013;2:e00308.

33. Zomber G, Reuveny S, Garti N, Shafferman A, Elhanany E. Effects of spontaneous deamidation on the cytotoxic activity of the Bacillus anthracis protective antigen. J Biol Chem. 2005;280:39897-906.

34. Jones RM, Burke M, Dubose D, Chichester JA, Manceva S, Horsey A, et al. Stability and pre-formulation development of a plant-produced anthrax vaccine candidate. Vaccine. 2017;35:5463-70.

35. Jiang G, Joshi SB, Peek LJ, Brandau DT, Huang J, Ferriter MS, et al. Anthrax vaccine powder formulations for nasal mucosal delivery. J Pharm Sci. 2006;95:80-96.

36. Mikszta JA, Sullivan VJ, Dean C, Waterston AM, Alarcon JB, Dekker JP 3rd, et al. Protective immunization against inhalational anthrax: a comparison of minimally invasive delivery platforms. J Infect Dis. 2005;191:278-88. 
37. Wang SH, Kirwan SM, Abraham SN, Staats HF, Hickey AJ. Stable dry powder formulation for nasal delivery of anthrax vaccine. J Pharm Sci. 2012b;101:31-47.

38. Illum L, Jabbal-Gill I, Hinchcliffe M, Fisher A, Davis S. Chitosan as a novel nasal delivery system for vaccines. Adv Drug Deliv Rev. 2001;51:81-96.

39. Kemble G, Greenberg H. Novel generations of influenza vaccines. Vaccine. 2003;21:1789-95.

40. Flick-Smith HC, Eyles JE, Hebdon R, Waters EL, Beedham RJ, Stagg TJ, et al. Mucosal or parenteral administration of microsphere-associated Bacillus anthracis protective antigen protects against anthrax infection in mice. Infect Immun. 2002;70:2022-8.

41. Lauring AS, Jones JO, Andino R. Rationalizing the development of live attenuated virus vaccines. Nat Biotechnol. 2010;28:573-9.

42. Burke, C.J., Hsu, T., Volkin, D.B., 1999. Formulation, stability, and delivery of live attenuated vaccines for human use. Critical Reviews $^{\mathrm{TM}}$ in Therapeutic Drug Carrier Systems 16:1-83.

43. Chen D, Kristensen D. Opportunities and challenges of developing thermostable vaccines. Expert Rev Vaccines. 2009;8:547-57.

44. Cleland JL, Powell MF, Shire SJ. The development of stable protein formulations: a close look at protein aggregation, deamidation, and oxidation. Crit Rev Ther Drug Carrier Syst. 1993;10:307-77.

45. Volkin DB, Mach H, Middaugh CR. Degradative covalent reactions important to protein stability. Mol Biotechnol. 1997;8:105-22.

46. Saboo S, Tumban E, Peabody J, Wafula D, Peabody DS, Chackerian B, et al. Optimized formulation of a thermostable spray-dried virus-like particle vaccine against human papillomavirus. Mol Pharm. 2016;13:1646-55.

47. Kraan H, van Herpen P, Kersten G, Amorij JP. Development of thermostable lyophilized inactivated polio vaccine. Pharm Res. 2014;31:2618-29.

48. Dorval BL, Chow M, Klibanov AM. Lysine and other diamines dramatically stabilize poliovirus against thermoinactivation. Biotechnol Bioeng. 1990;35:1051-4.

49. Ofori-Anyinam O, Vrijsen R, Kronenberger P, Boeye A. Heat stabilized, infectious poliovirus. Vaccine. 1995;13:983-6.

50. Crainic R, Wu R, Otelea D, Georgescu M, Delpeyroux F, Guillot $\mathrm{S}$, et al. The replacement of water with deuterium oxide significantly improves the thermal stability of the oral poliovirus vaccine. Dev Biol Stand. 1996;87:161-6.

51. Berge TO, Jewett RL, Blair WO. Preservation of enteroviruses by freeze drying. Appl Microbiol. 1971;22:850-3.

52. Ferreira E, Mendes YS, Silva JL, Galler R, Oliveira AC, Freire MS, et al. Effects of hydrostatic pressure on the stability and thermostability of poliovirus: a new method for vaccine preservation. Vaccine. 2009;27:5332-7.

53. Amorij JP, Huckriede A, Wilschut J, Frijlink HW, Hinrichs WL. Development of stable influenza vaccine powder formulations: challenges and possibilities. Pharm Res. 2008;25:125673.

54. Glezen WP. Serious morbidity and mortality associated with influenza epidemics. Epidemiol Rev. 1982;4:25-44.

55. Neuzil KM, Hohlbein C, Zhu Y. Illness among schoolchildren during influenza season: effect on school absenteeism, parental absenteeism from work, and secondary illness in families. Arch Pediatr Adolesc Med. 2002;156:986-91.

56. Wright PF, Webster RG. Orthomyxoviruses. In: Fields BN, Knipe DM, editors. Fields Virology, 4th ed. Philadelphia: Lippincott Williams \& Wilkins; 2001. p. 1533-1579.

57. Kamerzell TJ, Esfandiary R, Joshi SB, Middaugh CR, Volkin DB. Protein-excipient interactions: mechanisms and biophysical characterization applied to protein formulation development. Adv Drug Deliv Rev. 2011;63:1118-59.

58. White JA, Estrada M, Flood EA, Mahmood K, Dhere R, Chen D. Development of a stable liquid formulation of live attenuated influenza vaccine. Vaccine. 2016:34:3676-83.

59. World health Organization, 2006. Guidelines on stability evaluation of vaccines, WHO/BS/062049.
60. Ohtake S, Martin RA, Yee L, Chen D, Kristensen DD, Lechuga-Ballesteros $\mathrm{D}$, et al. Heat-stable measles vaccine produced by spray drying. Vaccine. 2010;28:1275-84.

61. Edens C, Collins ML, Ayers J, Rota PA, Prausnitz MR. Measles vaccination using a microneedle patch. Vaccine. 2013;31:3403-9.

62. Zhang J, Pritchard E, Hu X, Valentin T, Panilaitis B, Omenetto FG, et al. Stabilization of vaccines and antibiotics in silk and eliminating the cold chain. Proc Natl Acad Sci U S A. 2012;109:11981-6.

63. Ghobadloo SM, Balcerzak AK, Gargaun A, Muharemagic D, Mironov GG, Capicciotti CJ, et al. Carbohydrate-based ice recrystallization inhibitors increase infectivity and thermostability of viral vectors. Sci Rep. 2014;4:5903.

64. Nascimento I, Leite L. Recombinant vaccines and the development of new vaccine strategies. Braz J Med Biol Res. 2012;45:1102-11.

65. Michel ML, Tiollais P. Hepatitis B vaccines: protective efficacy and therapeutic potential. Pathol Biol (Paris). 2010;58:288-95.

66. Beutels P. Economic evaluations of hepatitis B immunization: a global review of recent studies (1994-2000). Health Econ. 2001;10:751-74.

67. Maynard JE, Kane MA, Hadler SC. Global control of hepatitis $B$ through vaccination: role of hepatitis $B$ vaccine in the expanded programme on immunization. Clin Infect Dis. 1989;11:S574-8.

68. Van Damme P, Cramm M, Safary A, Vandepapeliere P, Meheus A. Heat stability of a recombinant DNA hepatitis B vaccine. Vaccine. 1992;10:366-7.

69. Zapata MI, Feldkamp JR, Peck GE, White JL, Hem SL. Mechanism of freeze-thaw instability of aluminum hydroxycarbonate and magnesium hydroxide gels. J Pharm Sci. 1984;73:3-8.

70. Diminsky D, Moav N, Gorecki M, Barenholz Y. Physical, chemical and immunological stability of CHO-derived hepatitis B surface antigen (HBsAg) particles. Vaccine. 1999;18:3-17.

71. Brandau DT, Jones LS, Wiethoff CM, Rexroad J, Middaugh CR. Thermal stability of vaccines. J Pharm Sci. 2003;92:218-31.

72. Jezek J, Chen D, Watson L, Crawford J, Perkins S, Tyagi A, et al. A heat-stable hepatitis B vaccine formulation. Hum Vaccin. 2009;5:529-35.

73. Braun LJ, Jezek J, Peterson S, Tyagi A, Perkins S, Sylvester D, et al. Characterization of a thermostable hepatitis $\mathrm{B}$ vaccine formulation. Vaccine. 2009;27:4609-14.

74. Tonnis WF, Amorij JP, Vreeman MA, Frijlink HW, Kersten GF, Hinrichs WL. Improved storage stability and immunogenicity of hepatitis B vaccine after spray-freeze drying in presence of sugars. Eur J Pharm Sci. 2014;55:36-45.

75. Brotherton JM, Ogilvie GS. Current status of human papillomavirus vaccination. Curr Opin Oncol. 2015;27:399-404.

76. Govan VA. A novel vaccine for cervical cancer: quadrivalent human papillomavirus (types 6,11,16, and 18) recombinant vaccine (Gardasil ${ }^{\circledR}$ ). Ther Clin Risk Manag. 2008;4:65-70.

77. Pérez O, Batista-Duharte A, González E, Zayas C, Balboa J, Cuello $\mathrm{M}$, et al. Human prophylactic vaccine adjuvants and their determinant role in new vaccine formulations. Braz $\mathrm{J}$ Med Biol Res. 2012;45:681-92.

78. Shi L, Sanyal G, Ni A, Luo Z, Doshna S, Wang B, et al. Stabilization of human papillomavirus virus-like particles by non-ionic surfactants. J Pharm Sci. 2005;94:1538-51.

79. Fausch SC, Da Silva DM, Eiben GL, Le Poole IC, Kast WM. HPV protein/peptide vaccines: from animal models to clinical trials. Front Biosci. 2003;8:s81-91.

80. Gerard C, Baudson N, Kraemer K, Bruck C, Garcon N, Paterson Y, et al. Therapeutic potential of protein and adjuvant vaccinations on tumour growth. Vaccine. 2001;19:2583-9.

81. Hung CF, Ma B, Monie A, Tsen SW, Wu TC. Therapeutic human papillomavirus vaccines: current clinical trials and future directions. Expert Opin Biol Ther. 2008;8:421-39.

82. Bam NB, Cleland JL, Yang J, Manning MC, Carpenter JF, Kelley RF, et al. Tween protects recombinant human growth hormone against agitation-induced damage via hydrophobic interactions. J Pharm Sci. 1998;87:1554-9. 
83. Hageman M. Stability of protein pharmaceuticals. Part A. Chemical and physical pathways of protein degradation. In: Ahern TJ, Manning MC, editors. . New York: Plenum Press; 1994. p. 273-309.

84. Avis KE, Wu VL. Biotechnology and biopharmaceutical manufacturing, processing, and preservation. Boca Raton: CRC Press; 1996.

85. Avery OT, Goebel WF. Chemo-immunological studies on conjugated carbohydrate-proteins : II. Immunological specificity of synthetic sugar-protein antigens. J Exp Med. 1929;50:533-50.

86. Black S, Shinefield H, Fireman B, Lewis E, Ray P, Hansen JR, et al. Efficacy, safety and immunogenicity of heptavalent pneumococcal conjugate vaccine in children. Northern California Kaiser Permanente Vaccine Study Center Group. Pediatr Infect Dis J. 2000;19:187-95.

87. Finn A. Bacterial polysaccharide-protein conjugate vaccines. Br Med Bull. 2004;70:1-14.

88. Koskela M, Leinonen M, Haiva VM, Timonen M, Makela PH. First and second dose antibody responses to pneumococcal polysaccharide vaccine in infants. Pediatr Infect Dis. 1986;5:45-50.

89. Lindberg AA. Glycoprotein conjugate vaccines. Vaccine. 1999;17(Suppl 2):S28-36.

90. Peltola H, Kayhty H, Virtanen M, Makela PH. Prevention of Hemophilus influenzae type b bacteremic infections with the capsular polysaccharide vaccine. N Engl J Med. 1984;310:15616.

91. Richmond P, Borrow R, Findlow J, Martin S, Thornton C, Cartwright K, et al. Evaluation of De-O-acetylated meningococcal $\mathrm{C}$ polysaccharide-tetanus toxoid conjugate vaccine in infancy: reactogenicity, immunogenicity, immunologic priming, and bactericidal activity against O-acetylated and De-Oacetylated serogroup C strains. Infect Immun. 2001;69:237882.

92. Wang JF, Caugant DA, Morelli G, Koumare B, Achtman M. Antigenic and epidemiologic properties of the ET-37 complex of Neisseria meningitidis. J Infect Dis. 1993;167:1320-9.

93. Friedrich F. Rare adverse events associated with oral poliovirus vaccine in Brazil. Braz J Med Biol Res. 1997;30:695-703.

94. Minor P. Vaccine-derived poliovirus (VDPV): impact on poliomyelitis eradication. Vaccine. 2009;27:2649-52.

95. Salas-Peraza D, Avila-Agüero ML, Morice-Trejos A. Switching from OPV to IPV: are we behind the schedule in Latin America? Expert Rev Vaccines. 2010;9:475-83.

96. Atkinson W, Wolfe S, Hamborsky J. Epidemiology and prevention of vaccine-preventable diseases. Washington: Public Health Foundation; 2011.

97. Kristensen D. Summary of stability data for licensed vaccines. Seattle: PATH; 2012.

98. Qi W, Zeng Y, Orgel S, Francon A, Kim JH, Randolph TW, et al. Preformulation study of highly purified inactivated polio vaccine, serotype 3. J Pharm Sci. 2014;103:140-51.

99. Tzeng SY, Guarecuco R, McHugh KJ, Rose S, Rosenberg EM, Zeng Y, et al. Thermostabilization of inactivated polio vaccine in PLGA-based microspheres for pulsatile release. J Control Release. 2016;233:101-13.

100. Kraan H, Ploemen I, van de Wijdeven G, Que I, Lowik C, Kersten G, et al. Alternative delivery of a thermostable inactivated polio vaccine. Vaccine. 2015;33:2030-7.

101. Edwards KM, Meade BD, Decker MD, Reed MF, Rennels MB, Steinhoff MC, et al. Comparison of 13 acellular pertussis vaccines: overview and serologic response. Pediatrics. 1996;97:784.

102. Skibinski DA, Baudner BC, Singh M, O'Hagan DT. Combination vaccines. J Glob Infect Dis. 2011;3:63-72.

103. Jivapisarnpong T. Combined vaccines-case study. Biologicals. 2009;37:416.

104. Dobbelaer R, Pfleiderer M, Haase M, Griffiths E, Knezevic I, Merkle A, et al. Guidelines on stability evaluation of vaccines. Biologicals. 2009;37:424-34 discussion 421-423.

105. Eskola J, Ward J, Dagan R, Goldblatt D, Zepp F, Siegrist CA. Combined vaccination of Haemophilus influenzae type $\mathrm{b}$ conjugate and diphtheria-tetanus-pertussis containing acellular pertussis. Lancet. 1999;354:2063-8.

106. Schmitt, H.J., 1995. Immunogenicity and reactogenicity of two Haemophilus influenzae type b tetanus conjugate vaccines admninistered by reconstituting with diphtheria-tetanusacellular pertussis vaccine or given as separate injections (abstract G63), 35th Interscience Conference on Antimicrobial Agents and Chemotherapy, San Francisco.

107. Slack MH, Schapira D, Thwaites RJ, Burrage M, Southern J, Andrews $\mathrm{N}$, et al. Immune response of premature infants to meningococcal serogroup $\mathrm{C}$ and combined diphtheria-tetanus toxoids-acellular pertussis-Haemophilus influenzae type b conjugate vaccines. J Infect Dis. 2001;184:1617-20.

108. Decker MD. Principles of pediatric combination vaccines and practical issues related to use in clinical practice. Pediatr Infect Dis J. 2001;20:S10-8.

109. Schmitt HJ, Knuf M, Ortiz E, Sänger R, Uwamwezi MC, Kaufhold A. Primary vaccination of infants with diphtheriatetanus-acellular pertussis-hepatitis B virus-inactivated polio virus and Haemophilus influenzae type $\mathrm{b}$ vaccines given as either separate or mixed injections. J Pediatr. 2000;137:304-12.

110. Dagan R, Poolman JT, Zepp F. Combination vaccines containing DTPa-Hib: impact of IPV and coadministration of CRM197 conjugates. Expert Rev Vaccines. 2008;7:97-115.

111. Jatana SK, Nair M. Combination vaccines. Med J Armed Forces India. 2007;63:167-71.

112. Zeng Y, Fan H, Chiueh G, Pham B, Martin R, LechugaBallesteros D, et al. Towards development of stable formulations of a live attenuated bacterial vaccine: a preformulation study facilitated by a biophysical approach. Hum Vaccin. 2009;5:322-31.

113. Rao YU, William J, Kalyanaraman VR. A study of the stability of the pertussis component of diphtheria-tetanus-pertussis (DTP) vaccines. J Biol Stand. 1985;13:267-70.

114. Galazka A, Milstien J, Zaffran M. Thermostability of vaccines: global programme for vaccines and immunization. Geneva: World Health Organization; 1998. 